Pacific Journal of Mathematics

LOWER BOUNDS ON THE STABLE RANGE OF POLYNOMIAL 


\title{
LOWER BOUNDS ON THE STABLE RANGE OF POLYNOMIAL RINGS
}

Michael R. Gabel

\begin{abstract}
Using the existence of certain nonfree projective modules, lower bounds on the stable range of commutative polynomial rings are established.
\end{abstract}

1. Introduction. The stable range of a commutative ring $R$ is the largest integer $n$ such that there exists a unimodular sequence $r_{1}, \cdots, r_{n}$ in $R$ which is not stable. (See definitions 2.1 and 2.2.) Using the existence of certain nonfree projectives, we prove the following theorem concerning lower bounds for the stable range of polynomial rings:

THEOREM A. Let $K$ be any commutative ring. For every integer $n \geqq 1$ let $K(n)=K\left[Z_{1}, \cdots, Z_{n}\right]$ be the polynomial ring over $K$ in indeterminates $Z_{1}, \cdots, Z_{n}$. Then

(1) The stable range of $K(1)$ is at least 2

(2) The stable range of $K(n)$ is at least $[n / 2]+1$ if $n \geqq 2$.

(3) (Vasershtein, [6, Theorem 8]). If there is a ring homomorphism from $K$ to a subfield of real numbers, then the stable range of $K(n)$ is at least $n+1$.

The techniques of this paper are essentially the same as those in [2]. That paper contains a weaker form of Theorem $\mathrm{A}(2)([n / 2]$ appears in place of $[n / 2]+1$, and there are certain restrictions on the number of indeterminates.)

2. Basic definitions and propositions. Throughout this paper, all rings are commutative with identity and ring homomorphisms preserve the identity element.

Definition 2.1. A sequence $r_{1}, \cdots, r_{n}$ of elements in a ring $R$ is called unimodular if the ideal $\left(r_{1}, \cdots, r_{n}\right)$ is all of $R$.

DEFINITION 2.2. A unimodular sequence $r_{1}, \cdots, r_{n}$ of elements in a ring $R$ is called stable if $n \geqq 2$ and if there exist $b_{1}, \cdots, b_{n-1} \in R$ such that the sequence $r_{1}+b_{1} r_{n}, r_{2}+b_{2} r_{n}, \cdots, r_{n-1}+b_{n-1} r_{n}$ is again unimodular.

Proposition 2.3. Let $R$ be a ring and $I \subset R$ a proper ideal of $R$. Let $r_{1}, \cdots, r_{t}$ be a sequence of elements in $R$ such that their 
residues $\bar{r}_{1}, \cdots, \bar{r}_{t}$ form a unimodular sequence in $R / I$ which is not stable. Choose any $i \in I$ and $s_{1}, \cdots, s_{t} \in R$ such that $r_{1} s_{1}+\cdots+$ $r_{t} s_{t}+i=1$. Then the sequence $r_{1}, \cdots, r_{t-1}, r_{t} s_{t}+i$ is unimodular but not stable in $R$. Consequently, the stable range of $R$ bounds that of $R / I$.

Proof. The sequence $r_{1}, \cdots, r_{t-1}, r_{t} s_{t}+i$ is clearly unimodular. If it were stable, then there would exist $b_{1}, \cdots, b_{t-1} \in R$ such that

$$
r_{1}+b_{1}\left(r_{t} s_{t}+i\right), \cdots, r_{t-1}+b_{t-1}\left(r_{t} s_{t}+i\right)
$$

is unimodular. Going $\bmod I$, this contradicts our assumption that $\bar{r}_{1}, \cdots, \bar{r}_{t}$ is not stable in $R / I$.

Definition 2.4. If $r_{1}, \cdots, r_{t} \in R$, $\operatorname{ker}\left[r_{1} \cdots r_{t}\right]$ will denote the kernel of the map $R^{t} \rightarrow R$ whose matrix is $\left[r_{1} \cdots r_{t}\right]$.

The following proposition appears in [2].

Proposition 2.5. Let $R$ be a ring and let $r_{1}, \cdots, r_{t}$ be a unimodular sequence in $R$. Let $P=\operatorname{ker}\left[r_{1} \cdots r_{t}\right]$. If $r_{1}, \cdots, r_{t}$ is stable, then $P$ is a free $R$-module.

Proof. Since $r_{1}, \cdots, r_{t}$ is stable, it's easy to see that the matrix $\left[r_{1} \cdots r_{t}\right]$ can be transformed to $\beta=\left[\begin{array}{llll}1 & 0 & \cdots & 0\end{array}\right]$ via elementary transformations. Consequently $\operatorname{ker}\left[r_{1} \cdots r_{t}\right] \cong \operatorname{ker} \beta$. But $\operatorname{ker} \beta$ is clearly free.

Proposition 2.6. (1) (Estes-Ohm, [1].) If

$$
Z_{1}, \cdots, Z_{n}, \quad 1-\sum_{i=1}^{n} Z_{i}^{2}
$$

is stable in $K\left[Z_{1}, \cdots, Z_{n}\right]$, then $Z_{1}, \cdots, Z_{n+1}, 1-\sum_{i=1}^{n+1} Z_{1}^{2}$ is stable in $K\left[Z_{1}, \cdots, Z_{n+1}\right]$.

(2) If $X_{1}, \cdots, X_{s}, 1-\sum_{i=1}^{s} X_{i} Y_{i}$ is stable in $K\left[X_{1}, \cdots, X_{s}\right.$, $\left.Y_{1}, \cdots, Y_{s}\right]$, then $X_{1}, \cdots, X_{s+1}, 1-\sum_{i=1}^{s+1} X_{i} Y_{i}$ is stable in $K\left[X_{1}, \cdots, X_{s+1}\right.$, $\left.Y_{1}, \cdots, Y_{s+1}\right]$.

Proof. The proof we give here of (2) is essentially the same as the proof of (1) given in [1]. For any integer $t \geqq 1$, let $Q_{t}=$ $1-\sum_{\imath=1}^{t} X_{i} Y_{i}$. Now observe that if $a_{1}, \cdots, a_{s} \in K\left[X_{1}, \cdots, X_{s}\right.$, $\left.Y_{1}, \cdots, Y_{s}\right]$, we have the following inclusion of ideals in $K\left[X_{1}, \cdots, X_{s+1}\right.$, $\left.Y_{1}, \cdots Y_{s+1}\right]$ :

$$
\left(X_{1}+a_{1} Q_{s}, \cdots, X_{s}+a_{s} Q_{s}\right) \leqq\left(X_{1}+a_{1} Q_{s+1}, \cdots, X_{s}+a_{s} Q_{s+1}, X_{s+1}\right) .
$$


3. Proof of Theorem A. The proof of part (1) is easy, as $Z_{1}, 1-Z_{1}^{2}$ is never stable in $K(1)$. (Just $\bmod$ out $K$ by a prime ideal to reduce to the domain case, where it is trivial.)

We now prove part (2). By Proposition 2.3, we can assume $n$ is even, say $n=2 s$. Now write $K(n)=K\left[X_{1}, \cdots, X_{s}, Y_{1}, \cdots, Y_{s}\right]$. We shall show that the unimodular sequence $X_{1}, \cdots, X_{s}, 1-\sum_{i=1}^{s} X_{i} Y_{i}$ is not stable. Let $\underline{m}$ be a maximal ideal of $K$. If $X_{1}, \cdots X_{s}, 1-$ $\sum_{i=1}^{s} X_{i} Y_{i}$ were stable in $K(n)$, then the image of this sequence under the canonical map $K(n) \rightarrow K / \underline{m}(n)$ would remain stable. Thus we may assume $K$ is a field. By Proposition 2.6(2), we may also assume $s \geqq 8$. Let $R=K(n)[U, V]$, where $U$ and $V$ are indeterminates and let $f=X_{1} Y_{1}+\cdots+X_{s} Y_{s}+U V-1$. Let $T=R /(f)$. Note that the sequence of residues $\bar{X}_{1}, \cdots, \bar{X}_{s}, \bar{U}$ is unimodular in $T$. Since $s \geqq 8$, the $T$-module $\operatorname{ker}\left[\bar{X}_{1}, \cdots, \bar{X}_{s}, \bar{U}\right]$ is not free ([4], Cor. 6.3). So by Proposition 2.5, $\bar{X}_{1}, \cdots, \bar{X}_{s}, \bar{U}$ is not stable in $T$. Since $X_{1} Y_{1}+\cdots+X_{s} Y_{s}+U V+(-f)=1$, Proposition 2.3 shows that $X_{1}, \cdots, X_{s}, U V-f$ is not stable in $K(n)[U, V]$. But $U V-f=$ $1-\sum_{i=1}^{s} X_{i} Y_{i}$ is an element of $K(n)$. That is, we have the unimodular sequence $X_{1}, \cdots, X_{s}, 1-\sum_{i=1}^{s} X_{i} Y_{i}$ in $K(n)$ which is not stable in $K(n)[U, V]$. So certainly it is not stable in $K(n)$. This proves Theorem A(2).

The proof of Theorem A(3) is similar. We will show that the stable range of $K\left[Z_{1}, \cdots, Z_{n}\right]$ is at least $n+1$ by showing that the unimodular sequence $Z_{1}, \cdots, Z_{n}, 1-\sum_{r=1}^{n} Z_{i}^{2}$ is not stable. Since $K$ maps to the reals, it suffices to show this when $K$ equals the reals. By Proposition 2.6(1) we may also assume $n \geqq 8$. Now, let $R=$ $K(n)[U]$, where $U$ is an indeterminate, let $f=Z_{1}^{2}+\cdots+Z_{n}^{2}+U^{2}-1$, and set $T=R /(f)$. Since $n \geqq 8$, The $T$-module $\operatorname{ker}\left[\bar{Z}_{1}, \cdots, \bar{Z}_{n}, \bar{U}\right]$ is not free [5]. Propositions 2.3 and 2.5 imply that $Z_{1}, \cdots, Z_{n}, U^{2}-f$ is not stable in $K(n)[U]$. But the $U^{2}$ drops out so we get that $Z_{1}, \cdots, Z_{n}, 1-\sum_{i=1}^{n} Z_{i}^{2}$ is not stable in $K(n)$. This proves Theorem $\mathrm{A}(3)$.

4. Remarks. (1) For any ring $K$, let $P_{1, n}^{0}(K)$ be the module over $K_{1, n}^{0} \equiv K\left[Z_{1}, \cdots, Z_{n}\right] /\left(\sum_{\imath=1}^{n} Z_{\imath}^{2}-1\right)$ defined by

$$
P_{1, n}^{0}(K)=\operatorname{ker}\left[\bar{Z}_{1}, \cdots, \bar{Z}_{n}\right] \text {. }
$$

The proof of Theorem A(3) yields that if $Z_{1}, \cdots, Z_{n}, 1-\sum_{i=1}^{n} Z_{\imath}^{2}$ is stable in $K(n)$, then $P_{1, n+1}^{0}(K)$ is a free $K_{1, n+1}^{0}$-module. Assume now that $K$ is a field. Then it is well-known [1] that the stable range of $K(n)$ is less than or equal to $n+1$. So when $P_{1, n+1}^{0}(K)$ is not free, the stable range of $K(n)$ equals $n+1$. Note that there are cases when $P_{1, n+1}^{0}(K)$ is free $(K=$ reals, $n=7)$ and the stable range 
of $K(n)$ is still $n+1$ [6]. Moreover, if $K$ is a finite field $P_{1, n+1}^{0}(K)$ is free [3] and the stable range of $K(n)$ is less than $n+1$ (Vasershtein).

(2) The nonstable unimodular sequence $Z_{1}, \cdots, Z_{n}, 1-\sum_{i=1}^{n} Z_{i}^{2}$ that we obtained in the proof of Theorem $\mathrm{A}(3)$ is the same one Vasershtein discovered in [6].

\section{REFERENCES}

1. Dennis Estes and Jack $\mathrm{Ohm}$, Stable range in commutative rings, J. Algebra, 7 , No. 3 (1967), 343-362.

2. M. R. Gabel and A. V. Geramita, Stable range for matrices, J. Pure Appl. Algebra, 5 (1974), 97-112.

3. A. V. Germita, Projective modules and homogeneous forms (unpublished).

4. M. Raynaud, Modules projectifs universels, Invent. Math., 6 (1968), 1-26.

5. R. G. Swan, Vector bundles and projective modules, Trans. Amer. Math. Soc., 105 (1962), 264-277.

6. L. N. Vasershtein, Stable rank of rings and dimensionality of topological spaces, Functional Analysis and its Applications, 5 (1971), 102-110.

Received December 13, 1974.

Purdue University 


\section{PACIFIC JOURNAL OF MATHEMATICS}

\section{EDITORS}

RICHARD ARENS (Managing Editor) University of California

Los Angeles, California 90024

\section{R. A. BEAUMONT}

University of Washington Seattle, Washington 98105
J. DugundjI Department of Mathematics University of Southern Californı Los Angeles, California 90007

D. Gilbarg AND J. Milgram Stanford University Stanford, California 94305

\section{ASSOCIATE EDITORS}

E. F. BECKENBACH

B. H. NeUMANN

F. WOLF

K. YosHidA

\section{SUPPORTING INSTITUTIONS}

UNIVERSITY OF BRITISH COLUMBIA CALIFORNIA INSTITUTE OF TECHNOLOGY UNIVERSITY OF CALIFORNIA MONTANA STATE UNIVERSITY UNIVERSITY OF NEVADA NEW MEXICO STATE UNIVERSITY OREGON STATE UNIVERSITY UNIVERSITY OF OREGON OSAKA UNIVERSITY

\author{
UNIVERSITY OF SOUTHERN CALIFORNIA \\ STANFORD UNIVERSITY \\ UNIVERSITY OF TOKYO \\ UNIVERSITY OF UTAH \\ WASHINGTON STATE UNIVERSITY \\ UNIVERSITY OF WASHINGTON \\ AMERICAN MATHEMATICAL SOCIETY \\ NAVAL WEAPONS CENTER
}




\section{Pacific Journal of Mathematics}

\section{Vol. 61, No. $1 \quad$ November, 1975}

Jiří Adámek, V. Koubek and Věra Trnková, Sums of Boolean spaces represent every

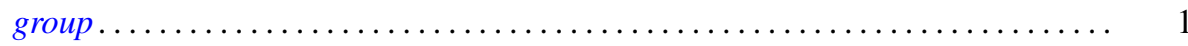

Richard Neal Ball, Full convex l-subgroups and the existence of $a^{*}$-closures of

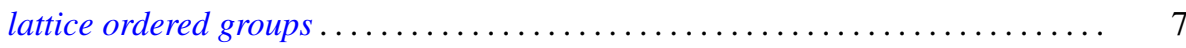

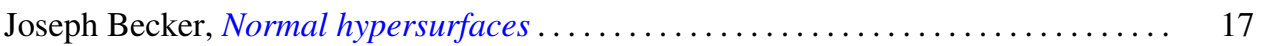

Gerald A. Beer, Starshaped sets and the Hausdorff metric . . . . . . . . . . . . . 21

Dennis Dale Berkey and Alan Cecil Lazer, Linear differential systems with

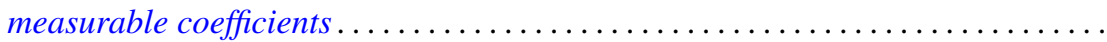

Harald Boehme, Glättungen von Abbildungen 3-dimensionaler

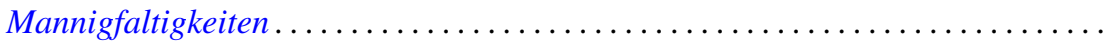

Stephen LaVern Campbell, Linear operators for which $T^{*} T$ and $T+T^{*}$

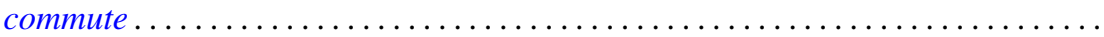

H. P. Dikshit and Arun Kumar, Absolute summability of Fourier series with

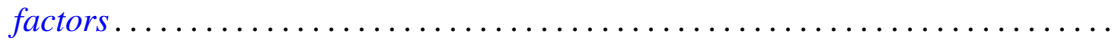

Andrew George Earnest and John Sollion Hsia, Spinor norms of local integral

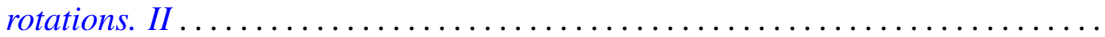

Erik Maurice Ellentuck, Semigroups, Horn sentences and isolic structures .........

Ingrid Fotino, Generalized convolution ring of arithmetic functions . . . . . . . . . . .

Michael Randy Gabel, Lower bounds on the stable range of polynomial rings .......

Fergus John Gaines, Kato-Taussky-Wielandt commutator relations and

characteristic curves

Theodore William Gamelin, The polynomial hulls of certain subsets of $C^{2}$

R. J. Gazik and Darrell Conley Kent, Coarse uniform convergence spaces. . .

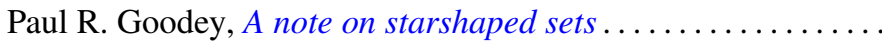

Eloise A. Hamann, On power-invariance

M. Jayachandran and M. Rajagopalan, Scattered compactification for $N \cup\{P\}$. . .

V. Karunakaran, Certain classes of regular univalent functions .

John Cronan Kieffer, A ratio limit theorem for a strongly subadditive set function in a locally compact amenable group .................

Siu Kwong Lo and Harald G. Niederreiter, Banach-Buck measure, density, and uniform distribution in rings of algebraic integers ........

Harold W. Martin, Contractibility of topological spaces onto metric spaces ....

Harold W. Martin, Local connectedness in developable spaces .

A. Meir and John W. Moon, Relations between packing and covering numbers of a tree.

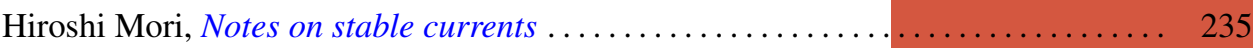

Donald J. Newman and I. J. Schoenberg, Splines and the logarithmic function . . . . 241

M. Ann Piech, Locality of the number of particles operator....

Fred Richman, The constructive theory of $K T$-modules .......

Gerard Sierksma, Carathéodory and Helly-numbers of

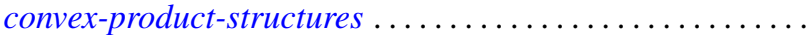

Raymond Earl Smithson, Subcontinuity for multifunctions .... . . 